\title{
Enteropathogenic Escherichia coli, Samonella, Shigella and Yersinia: cellular aspects of host- bacteria interactions in enteric diseases
}

Roberta Souza dos Reis ${ }^{1,2}$, Fabiana Horn ${ }^{1 *}$

\begin{abstract}
A successful infection of the human intestine by enteropathogenic bacteria depends on the ability of bacteria to attach and colonize the intestinal epithelium and, in some cases, to invade the host cell, survive intracellularly and disseminate from cell to cell. To accomplish these processes bacteria have evolved an arsenal of molecules that are mostly secreted by dedicated type III secretion systems, and that interact with the host, subverting normal cellular functions. Here we overview the most important molecular strategies developed by enteropathogenic Escherichia coli, Salmonella enterica, Shigella flexneri, and Yersinia enterocolitica to cause enteric infections. Despite having evolved different effectors, these four microorganisms share common host cellular targets.
\end{abstract}

\section{Introduction}

Diarrhea-causing pathogens employ a variety of sophisticated strategies to colonize the intestinal epithelium. In essence, ingested pathogens have evolved the abilities to: (1) resist non-specific host defenses, such as stomach acidity, peristalsis, mucosal cell exfoliation, intestinal mucins, and bacteriocins [1]; (2) adhere to intestinal epithelia; and (3) ultimately colonize the epithelia. Colonization may, or may not, involve cellular invasion. When cellular invasion occurs, it can be followed either by intracellular multiplication and spread of the bacteria to other tissues, or by bacterial persistence.

Although bacterial pathogens employ different strategies, they share common targets in the host cell and often cause the same cellular responses. Virulence factors act either from the extracellular milieu mimicking cell ligands, or are injected into the intracellular milieu, where they act in concert to manipulate signaling pathways, for example to subvert the plasticity of the cytoskeleton, interrupt the endocytic traffic, and, in some cases, mediate resistance to phagocytosis. The host cell, in turn, defends itself against infection by initiating an inflammatory response and by altering the intestinal fluid balance in order to extrude the unwanted bacteria,

\footnotetext{
* Correspondence: fabiana.horn@ufrgs.br

'Departamento de Biofísica, Universidade Federal do Rio Grande do Sul, P.O. Box 15005, 91501-970, Porto Alegre, Brazil
}

thus causing diarrhea. Hence, the success of infection depends on host cell-bacteria interactions, and in each step bacteria exploit the target cell machinery for their own benefit.

\section{Adhesion}

Adhesion is the preliminary step in the infectious process and requires strong and specific interactions. Table 1 lists the best characterized adhesins in the diarrheacausing bacteria discussed in this review, and their cognate host-cell receptors. Bacterial attraction to intestinal epithelia is also favored by local environmental factors such as alkaline milieu, humidity and anaerobiosis.

\section{Enteropathogenic Escherichia coli (EPEC)}

In response to the first contact with an epithelial cell, typical EPEC produce adhesive structures called bundleforming pili (BFP) and form microcolonies that increase bacterial resistance to host defenses and are involved in the localized adherence pattern [2] (Fig. 1a, upper panel). Colonization of the intestinal epithelia by EPEC also involves activation of the T3SS. Type III Secretion Systems are essential Gram-negative virulence determinants believed to mediate the transport of virulence factors from the bacterial cytoplasm into host cells. The four pathogens discussed in this review secrete their virulence factors through a T3SS. Each complex is composed of more than twenty proteins, including integral 
Table 1 Bacterial adhesive structures and their cognate receptors

\begin{tabular}{|c|c|c|c|c|}
\hline Pathogen & Adhesin & Receptor & Effect on host cell & Reference \\
\hline EPEC & $\begin{array}{l}\text { BFP } \\
\text { EspA } \\
\text { Intimin }\end{array}$ & $\begin{array}{l}\text { Not fully elucidated } \\
\text { Not fully elucidated } \\
\text { Tir }\end{array}$ & $\begin{array}{l}\text { Activation of } \mathrm{T}^{3 S S^{1}} \text { and formation of } \mathrm{A} / \mathrm{E}^{2} \text { lesions and changes of } \\
\text { epithelial cell morphology }\end{array}$ & {$[2,4]$} \\
\hline $\begin{array}{l}\text { Salmonella } \\
\text { sp. }\end{array}$ & $\begin{array}{l}\text { FimA } \\
\text { FimH }\end{array}$ & $\begin{array}{l}\text { Mannosylated proteins in } \\
\text { epithelial cells }\end{array}$ & $\begin{array}{l}\text { Activation of T3SS and transport of effector proteins necessary for } \\
\text { invasion }\end{array}$ & [14] \\
\hline Shigella sp. & $\begin{array}{l}\text { Complex } \\
\text { IpaB/C } \\
\text { IpaB }\end{array}$ & $\begin{array}{l}\text { Integrin } a_{5} b_{1} \\
\text { CD44 (natural receptor of } \\
\text { hyaluronic acid) }\end{array}$ & $\begin{array}{l}\text { Activation of T3SS and transport of effector proteins necessary for } \\
\text { invasion }\end{array}$ & {$[18,19]$} \\
\hline Yersinia sp. & $\begin{array}{l}\text { Invasin } \\
\text { YadA }\end{array}$ & $\begin{array}{l}b_{1} \text { integrins (natural receptor of } \\
\text { fibronectin) }\end{array}$ & $\begin{array}{l}\text { Activation of T3SS and transport of effector proteins necessary for } \\
\text { invasion and/or apoptosis }\end{array}$ & {$[44]$} \\
\hline
\end{tabular}

1. T3SS: Type III Secretion System.

2. A/E lesion: attaching/effacing lesion.

membrane proteins, chaperones and accessory proteins. They are divided into two substructures: a bacterial membrane-embedded base and an extracellular needlelike filament, often called a translocon, through which effector proteins are injected [3]. Transport of effector proteins from the bacterial cytoplasm to the periplasmic space is accomplished at the expense of ATP. Once in the host cell cytoplasm, these effector proteins interact with eukaryotic pathways to alter host cell signal transduction.

Most T3SS-secreted effector proteins are encoded by a pathogenicity island called locus of enterocyte effacement (LEE). EPEC T3SS translocon is constituted by EPEC secreted protein A (EspA), one of the first bacterial proteins secreted by the bacteria, that forms a filamentous extension from the bacterium to the surface of microvilli and acts in conjunction with EspB and EspD to form a pore structure in the host cell. The translocon permits translocation of other LEE effector proteins. The intimate adherence of EPEC - there is only $10 \mathrm{~nm}$ between bacterium and enterocyte - is promoted by an adhesin called intimin [2]. Intimin is an outer-membrane protein encoded by the eae gene in the LEE, and shares functional and structural homologies to the Yersinia pseudotuberculosis invasin protein $[4,5]$. The intimin receptor (Tir), also encoded by the LEE, is translocated by the T3SS and inserted into the host cell membrane, a dimer of Tir binds two intimin molecules [4]. Apart from its receptor function, Tir is phosphorylated on tyrosine residues by redundant host kinases [6], and this phosphorylation depends on Esps and T3SS [5]. Once phosphorylated, Tir binds Nck, a host protein that recruits and activates the amino-terminal domain of the Wiskott-Aldrich syndrome protein (N-WASP), which in turn recruits and activates actin-related protein $2 / 3$ (Arp2/3). This results in actin filament nucleation ${ }^{1}$ (see Appendix), leading to assembly of pedestal-like structures beneath the bacterium, typical of EPEC and enterohemorragic E. coli (EHEC), and formation of an attachment/effacement (A/E) lesion [7] (Fig. 1a, lower panel). Other effectors involved in microvilli effacement and pedestal formation are Map, EspF, EspG, EspH and EspZ, highlighting the complexity of these pathogenic processes [8]. Although the phosphorylation on tyrosine residues is sufficient for actin nucleation and lesion formation, Tir is also phophorylated on serine residues by host protein kinase A and this seems to be necessary for efficient pedestal formation [9]. Cholesterol in the host cell membrane also has a crucial function in EPEC adhesion, since cholesterol depletion reduces bacterial adherence and $\mathrm{A} / \mathrm{E}$ lesion formation [10].

The diarrhea observed in EPEC infections probably results from several mechanisms, including loss of absorptive surface due to microvilli effacement, increased intestinal permeability and active ion secretion. Nevertheless, it is not known if these mechanisms result from the action of EPEC virulence effectors or are consequences of host-cell responses to bacterial adherence [7]. A report indicates that EPEC is able, in a T3SS-dependent mechanism, to quickly inhibit in vitro sodium/glucose cotransporter (SGLT-1) activity even before microvilli effacement and pedestal formation. This is a key diarrheagenic mechanism, given that SGLT-1 is responsible for the daily uptake of approximately 6 liters of fluids from the intestine [8]. Another report, which employed the A/E pathogen Citrobacter rodentium in a mouse infection model, proposed that the localization of aquaporins (water channels) in colonocytes of the infected mice is altered during infection, in a process partially dependent on EspF and EspG [11]. For both EPEC and C. rodentium, there is growing evidence that these pathogens target and disrupt epithelial tight junctions, thus contributing to the loss of ions and water during diarrhea [12]. Moreover, using the $C$. rodentium mouse model, Higgins and colleagues (1999) [13] have shown that intimin plays a dual role in EPEC pathogenesis: in addition to its function as a cellular ligand, it drives a Th1 pro-inflammatory response 


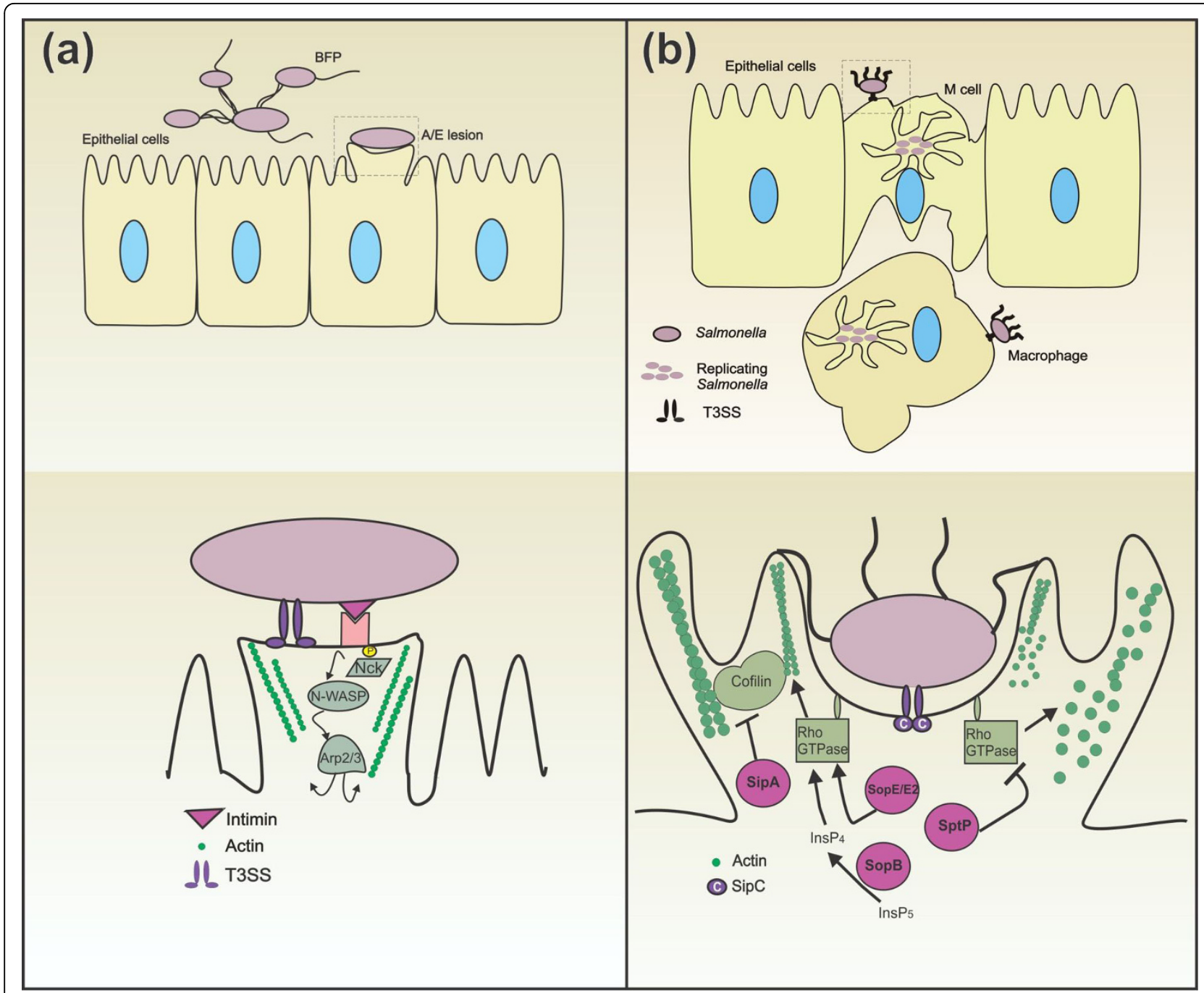

Figure 1 Pathogenic mechanisms of (a) enteropathogenic E. coli (EPEC) and (b) Salmonella enterica. (a) EPEC contact gut epithelial cells, produce BFP (upper panel), and activate their T3SS (lower panel). The bacterium translocates the receptor for its adhesin, intimin, called Tir, through the T3SS into the host cell cytoplasm; the interaction between intimin and Tir promotes tight adhesion. After being phosphorylated by host kinases, the Tir binds Nck, which activates N-WASP, which in turn activates Arp2/3, leading to actin nucleation and formation of a pedestal beneath the bacterium. (b) Salmonella interacts with M cells (upper panel), activates its T3SS and translocates SipA and SipC. SipC localizes to the plasma membrane, where it aids in the translocation of other Salmonella effectors and initiates actin nucleation. The C-terminal domain nucleates actin and the N-terminal domain of SipC bundles it, anchoring the resulting actin filaments to the cell surface below the bacterium. The injected SipA acts in synergy with SipC, as SipA binds to and stabilizes the F-actin filaments, and blocks the action of ADF/cofilin (lower panel). SopE1, SopE2 and SopB activate the RhoGTPases that regulate actin polymerization; SopE1 and SopE2 do so directly by acting as GEFs, and SopB indirectly by interfering with inositol phosphate metabolism. The activated RhoGTPases induce cytoskeletal rearrangements that result in bacterial uptake. SptP then switches off the RhoGTPases, and the eukaryotic cell regains its normal shape. Salmonella replicates inside the vacuole (upper panel).

resulting in mucosal thickening and crypt hyperplasia. Throughout the course of the infection, EPEC remain extracellular and the hyperplasia seems to offer sufficient area for new colonization.

\section{Salmonella enterica}

The initial interaction between Salmonella and the gut epithelium is believed to occur preferentially through $\mathrm{M}$ (Microfold) cells, either from Peyer patches or from solitary intestinal lymphoid tissue (SILT), which is scattered along the small intestine. $\mathrm{M}$ cells are follicle-associated epithelium (FAE) cells that function in antigen sampling; their basolateral surface is invaginated to form a pocket-like structure, to which macrophages and lymphocytes can migrate and where they interact with antigenic particles.

There is evidence that Salmonella sp. fimbriae play an important role in attachment to the gut mucosa. Type 1 
fimbriae are the most common adhesins in gram-negative bacteria; FimA and FimH are encoded by the fim operon, and the Salmonella genus contains at least another ten operons that encode fimbrins which, when expressed, act like adhesins [14]. Mutants in which genes involved in fimbrin biosynthesis were inactivated had their virulence attenuated [15]. Salmonella also contains non-fimbrial adhesins, encoded by chromosomal Salmonella pathogenicity island 4 (SPI-4), of which the best characterized so far is SiiE, implicated in invasion at the apical side of polarized epithelial cells [16].

\section{Shigella flexneri}

Very little is known about the adhesion structures that account for the initial contact between Shigella and a target cell. In contrast to other enteropathogenic bacteria that adhere to the small intestine, Shigella exhibits tropism for the colonic and rectal epithelia. This phenomenon could be explained by preferential adherence to human colonic mucin instead of small intestinal mucin observed in Shigella dysenteriae 1 [17]. Also, the fact that this binding was not inhibited by the presence of monosaccharides suggests that the receptor of Shigella adhesin is not a simple sugar.

In Shigella, the bacterial proteins responsible for adhesion are the same as those that initiate the process of invasion. Following adherence, Shigella initiate activation of T3SS and secretion of effector proteins into the host cell (Fig. 2a). The genes encoding the translocon itself and the effector proteins, including four bacterial chaperons, are located in a 31-kb pathogenicity island within a large virulence plasmid $(213 \mathrm{~kb})$.

The protein IpaB binds to the receptor for hyaluronic acid, CD44, while IpaB complexed with IpaC binds to the fibronectin receptor, $a_{5} b_{1}$ integrin $[18,19]$. The complex IpaB/C then forms a structure resembling a pore inserted into the host cell membrane [20]; the pore links the T3SS translocon to the eukaryotic cell cytoplasm, and allows rapid delivery of other effector proteins (Fig. 2a). Apparently, the binding of IpaB to cholesterol present in lipid rafts is required for pore formation [21]. Shigella attracts membrane rafts to its site of entry, and these rafts tend to harbor signaling proteins, many of which will be targeted by the bacterium. The effector IpaD localizes to the tip of the T3SS needle; it has been suggested that this protein is involved in recognition and contact with the host membrane and controls the release of other effectors [22].

\section{Yersinia enterocolitica}

After being ingested, Yersinia colonize the intestinal epithelium of the terminal portion of the ileum and proliferate in the underlying lymphoid tissue. Two non-fimbrial adhesins are crucial for adherence of Yersinia to host cells: invasin, encoded by the chromosomal gene inv, and YadA (Yersinia adhesin A), encoded by the Yersinia virulence plasmid (pYV). Invasin is an outer membrane protein that binds a subset of $b_{1}$ integrins $\left(a_{3} b_{1}\right.$, $a_{4} b_{1}, a_{5} b_{1}, a_{6} b_{1}$ and $a_{v} b_{1}$ ) through its extracellular, Cterminal, portion. Although invasin lacks an RGD domain and shares no sequence homology with fibronectin, the natural ligand of $b_{1}$ integrins, it binds $a_{5} b_{1}$ in the same residues and with a 100 -fold higher affinity than fibronectin [23]. While $b_{1}$ integrins are restricted to the basolateral surface of enterocytes [24], M cells express them on their apical surface, where they are available for bacterial adherence. The interaction of invasin with an integrin causes the agglomeration of integrin receptors over the bacteria-host interface, thus accounting for the "zipper" mechanism of Yersinia internalization observed by scanning electron microscopy. In contrast to invasin, YadA binds diverse extracellular matrix components, such as collagen, laminin and fibronectin, thus indirectly mediating integrin binding [25] (Fig. 2b).

\section{Cellular Invasion}

Following adhesion, infection by Salmonella, Shigella and Yersinia involves internalization, a process induced by the bacteria themselves. EPEC, in contrast, remain extracellular, although some reports point to an ability to invade due to the action of the effector protein EspG, which presumably interacts with tubulin [26], like the VirG protein of Shigella (see below).

In this section, we will consider the major mechanisms by which Salmonella, Shigella and Yersinia trigger their own uptake.

\section{Salmonella enterica}

Following adhesion between Salmonella and host epithelial cell or M cell, the T3SS encoded by chromosomal Salmonella pathogenicity island 1 (SPI-1) is activated. The bacterium then injects at least 12 effectors that trigger bacterial uptake.

Samonella inner proteins (Sip) A and C are probably the first proteins delivered through the T3SS to the host cell [27](Fig.1b). Within the host cell, SipC localizes to the plasma membrane, where it aids in the translocation of other Salmonella effectors and initiates actin nucleation. The C-terminal domain nucleates actin and the $\mathrm{N}$ terminal domain of SipC bundles it, anchoring the resulting actin filaments to the cell surface below the bacterium [28]. The injected SipA acts in synergy with SipC, as SipA binds to and stabilizes the F-actin filaments, and physically blocks the action of an actin depolymerising factor (ADF/cofilin).

The Salmonella outer proteins (Sop) E (SopE1 and SopE2), SopB and SptP are also delivered (in a SipCdependent way) through the T3SS translocon into the 


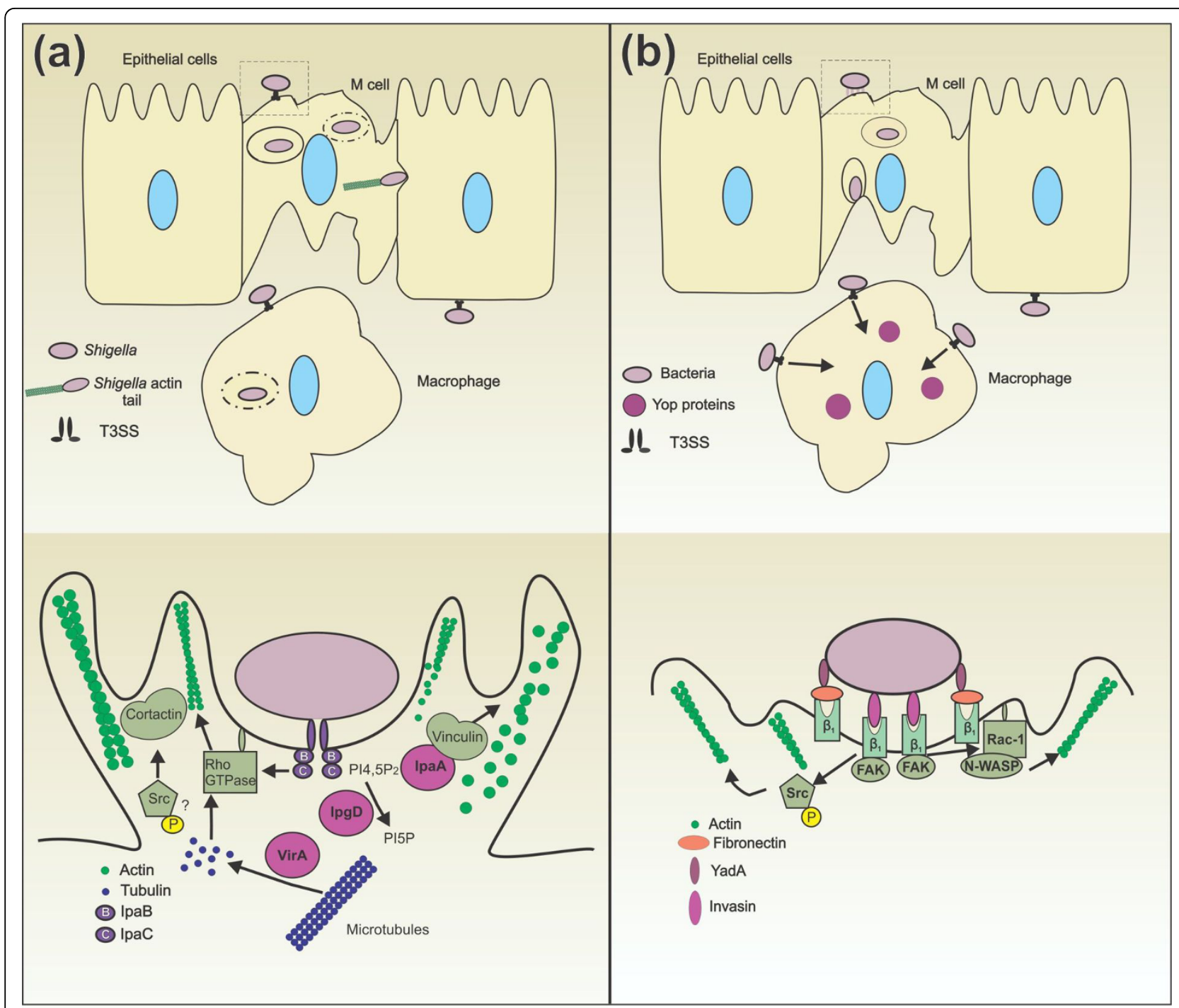

Figure 2 Pathogenic mechanisms of (a) Shigella flexneri and (b) Yersinia enterocolitica. (a) Shigella interacts preferentially with M cells in the colonic and rectal epithelia (upper panel), activates its T3SS and secretes IpaB and IpaC to form a pore inserted into the host cell membrane (lower panel). The bacterium then delivers effector proteins through the translocon to the eukaryotic cell cytoplasm. IpaC also activates RhoGTPases that, together with activated Src, recruit cortactin, involved in actin filament reorganization. VirA promotes microtubule destabilization, leading to activation of RhoGTPases. IpgD generates PI(5)P, thus promoting cell survival through Akt. IpaA binds to vinculin and induces actin depolymerization. Once inside the host cell, Shigella leaves the vacuole for the cytoplasm and escapes to neighboring cells (upper panel). (b) Yersinia adheres initially to $\mathrm{M}$ cells (upper panel) by means of its adhesins: invasin, which binds $\mathrm{b}_{1}$ integrins directly; and YadA, which binds $b_{1}$ integrins indirectly through fibronectin (lower panel). The interactions between the adhesins and integrins cause bacterial internalization following activation of FAK, Rac-1 and Src, which are involved in subtle actin cytoskeletal rearrangements. The vacuole containing the bacterium is transported towards the basolateral side of the $\mathrm{M}$ cell, where it is expelled into the dome region of the FAE (upper panel). When Yersinia interacts with phagocyte integrins, the T3SS is activated and a set of effector proteins (Yops) are translocated into the phagocyte cytoplasm. Yops are involved in the antiphagocytic and anti-inflammatory mechanisms used by Yersinia that lead to the formation of microabcesses in the FAE.

host cell, where they are targeted to the plasma membrane [27]. SopE and SopE2 act as guanine exchange factors (GEFs) for the Rho monomeric GTPases RhoG, Rac1 and Cdc42 [29], which are involved in the formation of filopodia extensions and lamellipodia structures. SopB (also known as SigD) also activates Cdc42, by a poorly understood mechanism, and RhoG by activating the endogenous GEF [30]. SopB is an inositol polyphosphate phosphatase that interferes with phosphoinositide phosphate and inositol phosphate metabolism [31]: SopB eliminates phosphatidylinositol3,5-biphosphate $\left(\operatorname{PtdIns}(3,5) \mathrm{P}_{2}\right), \operatorname{PtdIns}(4,5) \mathrm{P}_{2}$ and PtdIns $(3,4,5) \mathrm{P}_{3}$, generating PtdIns(3)P at the site of bacterial invasion; PtdIns(3)P is probably the activator of the GEF for RhoG, and is retained in the Salmonellacontaining vacuole [32]. 
Activation of RhoG, Rac1 and Cdc42 by Salmonella leads to activation of WASp, which, complexed with monomers of globular actin and Arp2/3, initiates nucleation of actin and its polymerization into actin filaments. The induced cytoskeletal rearrangements cause membrane ruffling and culminate in macropinocytosis of bacteria by the host epithelial cell [33]. The fact that Salmonella mutants defective in SopE, SopE2 or SopB remain invasive and only mutants lacking all three proteins display a non-invasive phenotype illustrates the redundancy of the Salmonella infection mechanisms [31].

Takeuchi (1967) had already observed that the epithelial cell soon recovers its normal shape following macropinocytosis of bacteria. This recovery is brought about by the effector protein SptP, which acts as a GTPase-activating factor for the RhoGTPases targeted by the SopE and SopB proteins, thereby switching off the signal [34].

\section{Shigella flexneri}

After having formed a pore in the membrane of $\mathrm{M}$ or epithelial cells, the IpaB/C complex triggers the initial events in actin polymerization. The C-terminal domain of IpaC activates Cdc42, which in turn activates Rac1, thereby inducing the formation of filopodial and lamellipodial extensions [35]. Shigella also somehow activates the tyrosine kinase Src (pp60c), which is implicated in recruitment by phosphorylation of actin-associated proteins such as cortactin [36].

The receptor for hyaluronic acid, CD44, once activated by IpaB, recruits ezrin to the site of entry. Ezrin recruitment seems to depend on Rho, whose activation is enhanced by the IpaB-CD44 complex [37]. Ezrin associates with $\mathrm{F}$-actin and functions as a membranecytoskeleton linker in the filopodial structures. Together, tyrosine kinases and small GTPases reorganize the actin cytoskeleton into entry structures similar to the focal adhesions in the host cell.

VirA is a protein encoded by a virulence plasmid and secreted in an Ipa-independent manner; it interacts with and destabilizes a/b-tubulin heterodimers, probably stimulating Rac-1 activity, which thus promotes the formation of lamellipodial structures around bacterial entry foci [38].

Two other T3SS-secreted proteins are IpgD and IpaA. IpgD is a phosphatase with homology to SopB/SigD of Salmonella; it generates PtdIns(5)P from PtdIns $(4,5) \mathrm{P}_{2}$ at the site of entry, and PtdIns(5)P in turn activates the PI-3 kinase/Akt pathway, thus contributing to cell survival [39]. IpaA complexes with the focal adhesion protein vinculin, and induces vinculin capping activity at the fast growing ends of $F$ actin, thus promoting $F$ actin depolymerization [40].

These events are summarized in Fig. 2a.

\section{Yersinia enterocolitica}

Activation of integrin receptors by invasin and/or YadA triggers several intracellular signals, which are very similar to the events that precede cell division: the cytoplasmic tail of b1 chain interacts with focal adhesion kinases (FAKs), Src and the Rac-1-Arp2/3 complex, which in turn trigger slight cytoskeleton rearrangements needed for bacterial uptake [41] (Fig. 2b, lower panel). This clustering or zipper mechanism of invasion contrasts with the trigger mechanism used by Salmonella and Shigella.

Yersinia species also hijack host cell phosphoinositide metabolism for their uptake. Rac-1 recruits, and Arf6 activates, the type I phosphatidylinositol-4-phosphate-5kinase (PtdIns(4) $\mathrm{P}(5) \mathrm{Ka})$, which forms $\operatorname{PtdIns}(4,5) \mathrm{P}_{2}$ at the entry site, where $\operatorname{Ptd} \operatorname{Ins}(4,5) \mathrm{P}_{2}$ may regulate phagocytic cup formation by coordinating membrane traffic and controlling F-actin production [42].

Yersinia internalized by $\mathrm{M}$ cells remains intracellular, bounded by a polymerized actin-coated vacuole. The bacterium survives inside the vacuole, even though it does not replicate. This vacuole is then transported from the apical to the basolateral side of the $M$ cell, where the bacterium is expelled and exposed to the dome region of the FAE, which is densely populated by dendritic cells, macrophages and lymphocytes (Fig. 2b, upper panel). In this way, Yersinia crosses the epithelial barrier. However, Yersinia species have evolved a dual strategy for avoiding destruction and establishing infection: an antiphagocytic and an anti-inflammatory strategy.

When Yersinia invasin interacts with phagocyte integrins, the extracellular adherent bacteria transfer a set of pathogenic factors, known as Yops (Yersinia outer proteins) through a pYv-encoded T3SS to the target cell, and this inhibits the uptake of Yersinia by interrupting the phagocytic pathway [43]. The T3SS apparatus is necessary for injection of the effectors (YopE, YopP, YopT, YopH, YopO and YopM) into the host cell [44]. Three translocator proteins are known to be required for the injection: YopB, YopD, which are believed to form a pore in the host cell membrane, and LcrV (also called V-antigen), which is localized to the tip of the needle and acts as a scaffold protein for the correct insertion of the pore formers in the membrane [45]. LcrV is also an important anti-inflammatory agent, which has been implicated in suppression of NF- $\kappa \mathrm{B}$ and interferon-g and the secretion of inhibitory interleukin IL-10 by macrophages [46].

YopH, YopE, YopT and YopO act indirectly on the actin cytoskeleton. YopH is a tyrosine phosphatase that dephosphorylates several macrophage proteins involved in focal adhesion, so opposing the phagocytic pathway induced by the invasin-integrin interaction [47]. 
Moreover, YopH rapidly blocks the neutrophil calcium signaling pathway induced by invasin-integrin binding, thereby inhibiting their degranulation [46]. YopE has GAP activity and there is evidence that it selectively inactivates Rac but not Rho or Cdc42 [48]. This inhibition of Rac activity ultimately leads to the arrest of membrane ruffling, otherwise an important step in engulfment. Aili and colleagues have shown that Yersinia devoid of YopE has high levels of Yop translocation into HeLa cells. This suggests that the GAP activity of YopE is also important in the modulation of pore formation, since YopE plays a role as a feedback inhibitor of Yop translocation [49]. YopT also has an anti-phagocytic role, since it removes the geranylgeranylated C-terminal cysteine of RhoA; once this occurs the enzyme is released from the plasma membrane and inactivated, so preventing actin reorganization [50]. The effector protein YopO, also known as YpkA (Yersinia protein kinase A), possesses a C-terminal actin-binding domain necessary for YopO activation, and a domain that mimics Rho and Rac guanine nucleotide dissociation inhibitors (GDI) [51] and maintains these factors in their GDP-bound inactive states, while its N-terminal region harbors a domain with serine/threonine kinase activity that phosphorylates a critical serine residue on $\mathrm{G}_{\mathrm{aq}}$ protein, blocking downstream calcium signaling [52]. Apparently, both the GDI and kinase activities of YopO are involved in disruption of the host cytoskeleton.

YopM, in contrast, has no enzymatic domain; instead it acts like a scaffolding protein, regulating the activity of two host cell kinases, PRK2 and RSK1, both involved in pathways signaling cell survival and proliferation. Association of YopM with PRK2 increases its kinase activity, which in turn activates RSK1. Once activated, the YopM-kinase complex may phosphorylate a still unknown substrate [53].

YopP, a homologue of YopJ, orchestrates an antiinflammatory process by exerting an inhibitory effect on both the mitogen-activated protein kinase (MAPK) and NF- $\kappa$ B signaling pathways [54]. YopJ has an acetyl transferase activity that modifies the activation loop of the MAPK and $\mathrm{I} \kappa \mathrm{b}$ kinases $^{2}$ (see Appendix), thereby preventing their phosphorylation and subsequent activation [55]. Inhibition of the MAPK and NF- $\kappa \mathrm{B}$ signaling pathways results in rapid apoptosis of the macrophages, which is important for establishing a systemic infection.

In addition to their action on cell adhesion, the surface factors YadA and Ail may be involved in serum resistance, probably by capturing the $\mathrm{C} 4 \mathrm{~b}$-binding protein that down-regulates the classical and lectin complement pathways [56]. Later in infection, when Yersinia interacts with epithelial cells, Yersinia invasin is able to stimulate NF- $\kappa$ B synthesis and trigger the production of pro-inflammatory cytokines, such as IL-8, via MAPK. The YadA-ECM- $b_{1}$ interaction also leads to IL- 8 production, although this occurs via a different MAPK [57].

IL-8 and other cytokines activated by Yersinia are chemoattractants and promoters of PMNs and macrophages. These cells are recruited to infection sites, leading to disruption of the epithelial barrier, and exposing the integrins localized on the basolateral sides of enterocytes, causing bacterial dissemination [57]. Ulceration and necrosis of the tissue subjacent to the FAE are also characteristic of Yersinia infections. Systemic dissemination begins 24-48 h after infection and may be enhanced by bacterial phagocytosis; the bacteria travel through the bloodstream towards the spleen and liver, where they replicate in microabscesses, forming microcolonies resistant to phagocytosis.

\section{Intracellular Lifestyle}

Here we will discuss how intracellular Salmonella and Shigella avoid being digested; while Salmonella remains in the vacuole and adapts to the harsh environment, Shigella escapes from the phagosome into the cytoplasm and neighboring cells.

\section{Salmonella enterica}

Once intracellular, either in M cells, epithelial cells or phagocytes, Salmonella is enclosed in an acidic compartment called a Salmonella-containing vacuole (SCV), in which the bacterium alters the endocytic pathway in order to avoid being destroyed, and replicates.

As mentioned above, Salmonella invasion is accompanied by the formation of PtdIns(3)P and disappearance of $\operatorname{PtdIns}(4,5) \mathrm{P}_{2}$ at the site of entry due to the action of SopB [32]. PtdIns $(4,5) \mathrm{P}_{2}$ depletion improves actin depolymerization and facilitates membrane fluidity. The local region of membrane invaginates to form PtdIns(3)Penriched vacuoles; PtdIns(3)P recruits early endosomal markers, including the GTPase Rab5 and EEA1 (early endosomal antigen 1), which promote fusion between the SCV and empty vesicles formed during bacterial invasion, creating a spacious habitat for Salmonella [32]. These early markers are quickly replaced by late endosomal markers (Rab7, LAMP-1, LAMP-2 and LAMP-3) and the vacuolar $\mathrm{H}^{+}$-ATPase (V-ATPase), which decreases the $\mathrm{pH}$ inside the SCV. Phagosome acidification by the V-ATPase is related to the maturation of lysosomes and the bactericidal function of lysosomal hydrolases. SCV acidification is a key stimulus for Salmonella to express and assemble, intracellularly, the second pathogenicity island-encoded T3SS (SPI-2 T3SS), approximately three hours after infection [58]. One study has revealed that SCV acidification in epithelial cells containing Salmonella occurs more slowly than in vacuoles containing heat-killed Salmonella or 
non-pathogenic Escherichia coli (expressing invasin). Apparently, this delay is independent of both Salmonella T3SSs, yet the delayed acidification seems to allow the bacteria to adapt to the new intracellular environment by decreasing the activity of lysosomal hydrolases [59].

According to a recent report by $\mathrm{Yu}$ and colleagues [60], the SPI-2 T3SS is assembled at the low $\mathrm{pH}$ of the $\mathrm{SCV}$, but secretion of a second set of effectors through the SCV membrane depends on sensing the neutral $\mathrm{pH}$ of the host cytosol. Effectors SseF and SseG colocalize with the microtubule cytoskeleton and interfere with its organization by inducing bundle formation. SseF and SseG, together with SifA (see below), affect the microtubule network and influence SCV positioning, helping to guide the Salmonella towards the perinuclear region, next to the Golgi complex [61,62]. This positioning facilitates interaction of the SCV with the endocytic and secretory pathways, allowing the pathogen to acquire extra membrane for expansion of the SCV, and nutrients to promote intracellular survival.

Four to six hours after entry, the SCV is characterized by the presence of long membrane structures, called Salmonella-induced filaments (Sifs) that extend from the SCV. Sifs are enriched in lysosomal membrane glycoproteins such as LAMP-1, and their formation is dependent on host cell microtubules [63]. These filaments increase the size of SCVs by incorporating into them PtdIns(3)P-enriched endosomes formed during Salmonella entry but devoid of bacteria, and thus provide space for Salmonella to replicate. SseF and SseG also somehow contribute to Sif formation, although to a lesser extend than SifA [64].

During its maturation, the SCV interacts with host endocytic-recycling regulators to regulate vacuole membrane renewal [65]. The lipid composition of the SCV may be modulated by the SPI-2 T3SS effector SseJ, which has a high degree of similarity to acyltransferases/ lipases and its enzymatic function is likely to oppose that of SifA, down-regulating Sif formation [66]. In addition, the effectors SspH2 and SseI were observed to colocalize with the F-actin cross-linkers filamin and profilin, and are thus apparently involved with the dynamics of the actin cytoskeleton around the SCV [67]. Actin filaments in the vicinity of the SCV membrane are assembled under the influence of the bacteria from within the vacuole in an SPI-2 T3SS-dependent manner, and seem to maintain the integrity of the vacuole and/ or recruit extra membrane that is necessary to increase the membrane surface area to enclose the replicating bacteria [68].

Inside the SCVs within phagocytes, the pathogen may inhibit, in an SPI-2-dependent manner, production of reactive oxygen species by preventing assembly of the NADPH oxidase complex [69], and of reactive nitrogen species by removing inducible nitric oxide synthase from the vicinity of the SCV [70]. It is possible that the actin meshwork and microtubule organization around the SCV affect the trafficking of these enzymes by physically blocking their colocalization with the vacuole.

Both SPI-1 and SPI-2 T3SSs expressed by Salmonella are able to induce macrophage death, although by distinct mechanisms and at different times of infection. The SPI-1 T3SS effector protein SipB activates caspase1 , triggering programmed pro-inflammatory cell death (pyroptosis) within 45 minutes of infection. Alternatively, macrophage death mediated by SipB may be simply a consequence of that effector's ability to induce cell lysis. Delayed macrophage death (up to $24 \mathrm{~h}$ ) may occur in an SPI-2 T3SS-dependent manner, although by a still unknown mechanism [71]. Once activated, caspase-1 cleaves the inactive precursors of the cytokines interleukin $1 \mathrm{~b}$ (IL-1b) and interleukin 18 (IL-18) to their mature, active forms. IL-1b and IL-18 are necessary to trigger the intestinal inflammation response characteristic of salmonellosis. Another pro-inflammatory cytokine generated during the infection is IL-8, activated by Cdc42 after it is activated by the SopE, SopE2 and/or SopB effectors [30].

The inflammatory response generated against Salmonella may compromise the intestinal barrier, allowing bacteria to cross it and encounter the phagocytes that have been attracted to the infection site. Once inside phagocytes, the bacteria survive and replicate, and some Salmonella species spread from Peyer's patches to the mesenteric lymph nodes, creating a systemic infection. Apoptosis of epithelial cells may also contribute to the observed disintegration of the intestinal epithelia in salmonellosis, since HT-29 human intestinal cells infected with $S$. dublin undergo delayed (not earlier than $24 \mathrm{~h}$ p.i.) apoptosis preceded by caspase- 3 activation, in a process dependent on effectors encoded by the SPI-2 loci [72].

Although efficient colonization of the host is advantageous for the pathogen, Salmonella appears to express antivirulence modulators during systemic infection: using a murine model, Gal-Mor et al. (2008) [73] have shown that when Salmonella Typhimurium reaches the intestinal tract, it expresses ZirT, an outer membrane protein with a b-barrel conformation, which translocates the protein ZirS; both proteins have been implicated in reducing the bacterial load in the infected mice.

\section{Shigella flexneri}

Once internalized by M cells of the FAE, Shigella, unlike Salmonella, disrupts the vacuole membrane in a process dependent on the IpaB and IpaC invasins and escapes into the host cell cytoplasm, where it proliferates. Once present in the cytoplasm, the pathogen accumulates 
polymerized actin at one pole of the bacterium, forming a "tail" that propels it through the cytoplasm and enables it to invade neighboring epithelial cells. The polymerization of actin depends on the action of IcsA (intracellular spread A)/VirG, an outer membrane protein that has a polar distribution on the bacterial surface.

IcsA/VirG recruits host cell cytoskeletal proteins; it interacts with N-WASP and increases its affinity for Arp2/3, forming the complex IcsA/N-WASP/Arp $2 / 3$ that induces actin nucleation. IcsA/VirG also recruits vinculin, and although vinculin is not necessary for movement of the pathogen, it may contribute to the polymerization of actin. Moreover, actin depolymerization factor (ADF)/cofilin, capping proteins and profilin are recruited to stabilize the tail. Shigella intracellular movement also depends on the tubulin-degrading activity of VirA, which opens the way through the microtubule network for Shigella [38]. When the bacterium eventually reaches the inner face of the basolateral membrane of the host cell, a finger-like protrusion is formed which is endocytosed by a neighbouring epithelial cell. Once endocytosed, the bacterium again escapes from the vacuole, proliferates in the host cell cytoplasm and invades new cells, thus advancing the course of disease. This process damages the tight junctions between adjacent cells, damage that is worsened by altered expression of tight junction-associated proteins induced by the pathogen [12]. Disruption of the integrity of the colonic mucosa leads to massive invasion of bacterial cells by exposing the basolateral sides of colonocytes.

As a consequence of the tissue invasion, epithelial cells start to produce a set of pro-inflammatory cytokines such as IL-8, which together with the infecting Shigella, strongly attracts polymorphonuclear (PMN) cells from the sub-epithelial layer to the intestinal lumen. The PMN infiltrate may exacerbate bacterial invasion at an early stage, but it ultimately leads to resolution of the infection, since the PMN cells do not allow the pathogen to cross the FAE and spread into the bloodstream. The negative consequences of PMN infiltration are severe inflammation and massive destruction of the colonic mucosa, causing mucosal abscesses and ulcers, the clinical characteristics of shigellosis [74].

After traversing M cells, Shigella exposes itself to an area of the FAE intensively populated with macrophages and dendritic cells. There, like Salmonella, it triggers macrophage pyroptosis: the translocated IpaB activates caspase1 , which in turn cleaves IL-1b and IL-18 to their mature, active forms [75]. Besides requiring membrane cholesterol [76], activation of caspase-1 by IpaB appears to require Ipaf, a Nod-like receptor localized on plasma and endosomal membranes and implicated in bacterial recognition [77]. IL-1b release causes rupture of the epithelial barrier, enhancing bacterial dissemination, which in turn increases inflammation and subsequent tissue destruction. Conversely, the parallel release of IL-18, a potent interferon-g inducer, aids host killing of Shigella by activated immune cells, thus demonstrating a protective role in shigellosis [78]. Shigella also secrete effectors through the T3SS that are engaged in down-regulating inflammation and these effectors may facilitate the early steps of infection. IpaH and OspF accumulate in the nucleus: IpaH binds to U2AF, a mammalian splicing factor, affecting the expression of pro-inflammatory cytokines [79], and OspF dephosphorilates and inactivates MAPKs [80]. A third effector, OspG, seems to prevent $\mathrm{I} \kappa \mathrm{Ba}$ degradation, so interfering with the NF- $\kappa \mathrm{B}$ activation pathway.

\section{Conclusions}

Knowledge of the pathogenesis of diarrheal diseases due to bacteria has grown greatly over the past two decades. The study of the bacterial pathogens described in this review has greatly illustrated the sophisticated mechanisms pathogens have developed to efficiently mimic and manipulate several host signaling pathways. In consequence, they succeed in colonizing the gut epithelium: EPEC do so by adhering tightly to epithelial cells and remaining extracellular; Salmonella, Shigella and Yersinia, in contrast, invade the gut epithelium. While Salmonella and Shigella are phagocytosed by macrophages and provoke an inflammatory response that disrupts the epithelial barrier, Yersinia avoids macrophage phagocytosis and inhibits the host inflammatory response. In all cases, the bacteria multiply and are able to leave the host and be transmitted to another host. Understanding at the molecular level of the systems developed by pathogenic bacteria may allow us to target essential bacterial effectors in novel therapeutic approaches to the infections. On the other hand, the lack of epidemiological studies identifying the predominant strain in a given endemic region, and the fact that diarrheal diseases are not always caused by a single etiological agent, certainly make such approaches more difficult to put into practice.

Regardless of its direct clinical application, the study of diarrheagenic bacteria as models of longstanding host-pathogen interactions has revealed how eukaryotic cells react under stress and how they mount an inflammatory response; such knowledge contributes to our understanding of infectious diseases, and also of noninfectious diseases. Moreover, the study of the diarrheagenic bacteria reviewed here has been giving new approaches in the investigation of cellular microbiology of other bacterial pathogens.

\section{Appendix}

1. Actin filaments (F-actin) are polymers of globular actin (G-actin) that associate with other proteins to 
form a cellular cortex beneath the plasma membrane. This net provides mechanical support for the cell membrane and allows changes that generate lamellipodia, filopodia and focal adhesion structures controlled by different members of the Rho GTPase family.

2. The family of NF-kB transcription factors is controlled by the binding of an inhibitor protein, named $\mathrm{IkB}$, which sequesters monomers of NF-kB in the cytoplasm. NF-kB activation requires removal of the $I k B$ from the complex through the action of an IkB kinase that phosphorylates the regulatory region of the inhibitor. The phosphorylated IkB conjugates with ubiquitin and is subsequently proteolytically degraded. Dimeric NF-kB is then able to move to the nucleus and bind to target DNA, thereby controlling the expression of a wide range of genes involved in the inflammatory response.

\section{Acknowledgements}

We would like to thank Julian Gross for help with the English writing and Roberto M.S. dos Reis for assistance with the figures.

\section{Author details}

'Departamento de Biofísica, Universidade Federal do Rio Grande do Sul, P.O. Box 15005, 91501-970, Porto Alegre, Brazil. ²Departamento de Microbiologia, Universidade Federal do Rio Grande do Sul, Av. Sarmento Leite, 500, 90050170, Porto Alegre, Brazil.

\section{Authors' contributions}

RSR draft the manuscript; RSR and FH wrote and revised the manuscript; both authors read and approved the final manuscript.

\section{Competing interests}

The authors declare that they have no competing interests.

Received: 1 June 2010 Accepted: 22 July 2010 Published: 22 July 2010

\section{References}

1. Moal VL, Servin AL: The front line of enteric host defense against unwelcome intrusion of harmful microorganisms: mucins, antimicrobial peptides, and microbiota. Clin Microbiol Rev 2006, 19:315-337.

2. Cleary J, Lai L, Shaw RK, Straatman-Iwanowska A, Donnenberg MS, Frankel G, Knutton S: Enteropathogenic Escherichia coli (EPEC) adhesion to intestinal epithelial cells: role of bundle-forming pili (BFP), EspA filaments and intimin. Microbiol 2004, 150:527-538.

3. Marlovits TC, Kubori T, Sukhan DR, Thomas DR, Galán JE, Unger VM: Structural insights into the assembly of the type III secretion needle complex. Science 2004, 306:1040-1042.

4. Luo Y, Frey EA, Pfuetzner RA, Creagh AL, Knoechel DG, Haynes CA, Finlay BB, Strynadka NCJ: Crystal structure of enteropathogenic Escherichia coli intimin-receptor complex. Nature 2000, 405:1073-1077.

5. Liu H, Magoun L, Luperchio S, Schauer DB, Leong JM: The Tir-binding region of enterohaemorrhagic Escherichia coli intimin is sufficient to trigger actin condensation after bacterial-induced host cell signaling. Mol Microbiol 1999, 34:67-81.

6. Bommarius B, Maxwell D, Swimm A, Leung S, Corbett A, Bornmann W, Kalman D: Enteropathogenic Escherichia coli Tir is an $\mathrm{SH} 2 / 3$ ligand that recruits and activates tyrosine kinases required for pedestal formation. Mol Microbiol 2007, 63:1748-1768.

7. Torres AG, Zhou X, Kaper JB: Adherence of diarrheagenic Escherichia coli strains to epithelial cells. Infect immun 2005, 73:18-29.

8. Dean P, Maresca M, Schüller S, Phillips AD, Kenny B: Potent diarrheagenic mechanism mediated by the cooperative action of three enteropathogenic Escherichia coli-injected effector proteins. Proc Natl Acad Sci USA 2005, 103:1876-1881.

9. Warawa J, Kenny B: Phosphoserine modification of the enteropathogenic Escherichia coli Tir molecule is required to trigger conformational changes in Tir and efficient pedestal elongation. Mol Microbiol 2001, 42:1269-1280.

10. Riff JD, Callahan JW, Sherman PM: Cholesterol-enriched membrane microdomains are required for inducing host cell cytoskeletal rearrangements in response to attaching-effacing Escherichia coli. Infect Immun 2005, 73:7113-7125.

11. Guttman JA, Samji FN, Li Y, Deng W, Lin A, Finlay BB: Aquaporins contribute to diarrhea caused by attaching and effacing bacterial pathogens. Cell Microbiol 2007, 9:131-141.

12. Guttman JA, Finlay BB: Subcellular alterations that lead to diarrhea during bacterial pathogenesis. Trends Microbiol 2008, 16:535-542.

13. Higgins LM, Frankel G, Connerton I, Gonçalves NS, Dougan G, MacDonald T: Role of bacterial intimin in colonic hyperplasia and inflammation. Science 1999, 285:588-591.

14. Kisiela D, Laskowska A, Sapeta A, Kuczkowski M, Wieliczko A, Ugorski M: Functional characterization of the FimH adhesion from Salmonella enterica serovar Enteritidis. Microbiology 2006, 152:1337-1346.

15. Van der Velden AWM, Bäumler AJ, Tsolis RM, Hefron F: Multiple fimbrial adhesins are required for full virulence of Salmonella Typhimurium in mice. Infect Immun 1998, 66:2803-2808.

16. Gerlach RG, Claudio N, Rohde M, Jackel D, Wagner C, Hensel M: Cooperation of Salmonella pathogenicity islands 1 and 4 is required to breach epithelial barriers. Cell Microbiol 2008, 10:2364-2376.

17. Sudha P, Devaraj H, Devaraj N: Adherence of Shigella dysenteriae 1 to human colonic mucin. Curr Microbiol 2001, 42:381-387.

18. Watarai M, Funato S, Sasakawa C: Interaction of Ipa proteins of Shigella flexneri with $a_{5} b_{1}$ integrin promotes entry of the bacteria into mammalian cells. J Exp Med 1996, 183:991-999.

19. Lafont F, Van Nhieu GT, Hanada K, Sansonetti PJ, van der Goot FG: Initial steps of Shigella infection depend on the cholesterol/sphingolipid raftmediated CD44-IpaB interaction. EMBO Journal 2002, 21:4449-4457.

20. Blocker A, Gounon P, Larquet E, Niebuhr K, Cabiaux V, Parsot C, Sansonetti P: The tripartite Type III Secreton of Shigella flexneri inserts IpaB and IpaC into host membranes. J Cell Biol 1999, 14:7683-693.

21. Hayward RD, Cain RJ, McGhie EJ, Phillips N, Garner MJ, Koronakis V: Cholesterol binding by the bacterial type III translocon is essential for virulence effector delivery into mammalian cells. Mol Microbiol 2005, 56:590-603.

22. Espina M, Olive AJ, Kenjale R, Moore DS, Ausar SF, Kaminski RW, Oaks EV, Middaugh CR, Picking WD, Picking WL: IpaD localizes to the tip of the Type III Secretion System needle of Shigella flexneri. Infect Immun 2006, 74:4391-4400.

23. Hamburger ZA, Brown MS, Isberg RR, Bjorkman PJ: Crystal structure of invasin: a bacterial integrin-binding protein. Science 1999, 286:291-295.

24. Hamzaoui N, Kernéis S, Caliot E, Pringault E: Expression and distribution of $b_{1}$ integrins in in vitro-induced $M$ cells: implications for Yersinia adhesion to Peyer's patch epithelium. Cell Microbiol 2004, 6:817-828.

25. Hudson KJ, Bliska JB, Bouton AH: Distinct mechanisms of integrin binding by Yersinia pseudotuberculosis adhesins determine the phagocytic response of host macrophages. Cell Microbiol 2005, 7:1474-1489.

26. Hardwidge PR, Deng WY, Vallance BA, Rodriguez-Escudero I, Cid VJ, Molina M, Finlay BB: Modulation of host cytoskeleton function by the enteropathogenic Escherichia coli and Citrobacter rodentium effector protein EspG. Infect Immun 2005, 73:2586-2594.

27. Cain RJ, Hayward RD, Koronakis V: Deciphering interplay between Salmonella invasion effectors. PLoS Pathogens 2008, 4:e1000037.

28. Chang J, Chen J, Zhou D: Delineation and characterization of the actin nucleation and effector translocation activities of Salmonella SipC. Molecular Microbiology 2005, 55:1379-1389.

29. Friebel A, Ilchmann H, Aepfelbacher M, Ehrbar K, Machleidt W, Hardt WD: SopE and SopE2 from Salmonella typhimurium activate different sets of RhoGTPases of the host cell. J Biol Chem 2001, 276:34035-34040.

30. Patel JC, Galán JE: Differential activation and function of Rho GTPases during Salmonella-host cell interactions. J Cell Biol 2006, 175:453-63.

31. Zhou D, Chen LM, Hernandez L, Shears SB, Galán JE: Salmonella inositol polyphosphatase acts in conjunction with other bacterial effectors to 
promote host cell actin cytoskeleton rearrangements and bacterial internalization. Mol Microbiol 2001, 39:248-260.

32. Hernandez LD, Hueffer K, Wenk MR, Galán JE: Salmonella modulates vesicular traffic by altering phosphoinositide metabolism. Science 2004, 304:1805-1807.

33. Takeuchi A: Electron microscope studies of experimental Salmonella infection. Penetration into the intestinal epithelium by Salmonella Typhimurium. Am J Pathol 1967, 50:109-36.

34. Fu Y, Galán JE: A Salmonella protein antagonizes Rac- 1 and $\mathrm{Cdc} 42$ to mediate host cell recovery. Nature 1999, 401:293-7.

35. Van Nhieu GT, Caron E, Hall A, Sansonetti PJ: IpaC induces actin polymerization and filopodia formation during Shigella entry into epithelial cells. EMBO J 1999, 18:3249-3262.

36. Dumenil G, Olivo JC, Pellegrini S, Fellous M, Sansonetti PJ, Van Nhieu GT: Interferon K inhibits a Src-mediated pathway necessary for Shigellainduced cytoskeletal rearrangements in epithelial cells. J Cell Biol 1998, 143:1-10.

37. Skoudy A, Nhieu GT, Mantis N, Arpin M, Mounier J, Gounon P, Sansonetti PJ: A functional role for ezrin during Shigella flexneri entry into epithelial cells. J Cell Sci 1999, 112:2059-2068.

38. Yoshida S, Handa Y, Suzuki T, Ogawa M, Suzuki M, Tamai A, Abe A, Katayama E, Sasakawa C: Microtubule-severing activity of Shigella is pivotal for intercellular spreading. Science 2006, 314:985-989.

39. Pendaries $C$, Tronchère H, Arbibe L, Mounier J, Gozani O, Cantley L, Fry MJ, Gaits-lacovoni F, Sansonetti PJ, Payrastre B: Ptdlns(5)P activates the host cell PI3-kinase/akt pathway during Shigella flexneri infection. EMBO J 2006, 25:1024-1034.

40. Ramarao N, Le Clainche C, Izard T, Bourdet-Sicard R, Ageron E, Sansonetti PJ, Carlier M, Van Nhieu GT: Capping host actin filaments by vinculin activated by the Shigella IpaA carboxyl-terminal domain. FEBS Lett 2007, 581:853-857.

41. Eitel J, Heise T, Thiesen U, Dersch P: Cell invasion and IL-8 production pathways initiated by YadA of Yersinia pseudotuberculosis requires common signaling molecules (FAK, c-Src, Ras) and distinct cell factors. Cell Microbiol 2005, 7:63-77.

42. Wong KW, Isberg RR: Arf6 and phosphoinositol-4-phosphate-5-kinase activities permit bypass of the Rac1 requirement for b1 integrinmediated bacterial uptake. J Exp Med 2003, 198:603-614.

43. Heesemann J, Sing A, Trülzsch K: Yersinia's stratagem: targeting innate and adaptive immune defense. Curr Opin Microbiol 2006, 9:55-61.

44. Matsumoto H, Young GM: Translocated effectors of Yersinia. Curr Op Microbiol 2009, 12:94-100.

45. Broz P, Mueller CA, Müller SA, Philippsen A, Sorg I, Engel A, Cornelis GR: Function and molecular architecture of the Yersinia injectisome tip complex. Mol Microbiol 2007, 65:1311-1320.

46. Brubaker RR: Interleukin-10 and inhibition of innate immunity to Yersiniae: Roles of Yops and LcrV (V Antigen). Infect Immun 2003, 71:3673-3681

47. Grosdent N, Maridonneau-Parini I, Sory MP, Cornelis GR: Role of Yops and adhesins in resistance of Yersinia enterocolitica to phagocytosis. Infect Immun 2002, 70:4165-4176.

48. Andor A, Trülzsch K, Essler M, Roggenkamp A, Wiedemann A, Heesemann J, Aepfelbacher M: YopE of Yersinia, a GAP for Rho GTPases, selectively modulates Rac-dependent actin structures in endothelial cells. Cell Microbiol 2001, 3:301-310

49. Aili M, Isaksson EL, Carlsson SE, Wolf-Watz H, Rosqvist R, Francis MS: Regulation of Yersinia Yop-effector delivery by translocated YopE. Int J Med Microbiol 2007, 298:183-192.

50. Zumbihl R, Aepfelbacher M, Andor A, Jacobi CA, Ruckdeschel K, Rouot B, Heesemann J: The cytotoxin YopT of Yersinia enterocolitica induces modification and cellular redistribution of the small GTP-binding protein RhoA. J Biol Chem 1999, 274:29289-29293.

51. Prehna G, Ivanov MI, Bliska JB, Stebbins CE: Yersinia virulence depends on mimicry of host Rho-family nucleotide dissociation inhibitors. Cell 2006, 126:869-880.

52. Navarro L, Koller A, Nordfelth R, Wolf-Watz H, Taylor S, Dixon JE: Identification of a molecular target for the Yersinia protein kinase $\mathrm{A}$. $\mathrm{Mol}$ Cell 2007, 26:465-477.

53. McDonald C, Vacratsis PO, Bliska JB, Dixon JE: The Yersinia virulence factor YopM forms a novel protein complex with two cellular kinases. J Biol Chem 2003, 278:18514-18523.
54. Zhou H, Monack DM, Kayagaki N, Wertz I, Yin J, Wolf B, Dixit VM: Yersinia virulence factor YopJ acts as a deubiquitinase to inhibit NF- $\kappa$ B activation. J Exp Med 2005, 202:1327-1332.

55. Mittal R, Peak-Chew SY, McMahon HT: Acetylation of MEK2 and $I \kappa B$ kinase (IKK) activation loop residues by YopJ inhibits signaling. Proc Natl Acad Sci USA 2006, 103:18574-18579.

56. Kirjavainen V, Jarva H, Biedzka-Sarek M, Blom AM, Skurnik M, Meri S: Yersinia enterocolitica serum resistance proteins YadA and Ail bind the complement regulator C4b-binding protein. PLOS Path 2008, 4:e1000140.

57. Schmid Y, Grassl GA, Bühler OT, Skurnik M, Autenrieth IB, Bohn E: Yersinia enterocolitica adhesion A induces production of interleukin-8 in epithelial cells. Infect Immun 2004, 72:6780-6789.

58. Haraga A, Ohlson MB, Miller SI: Salmonellae interplay with host cells. Nat Rev Microbiol 2008, 6:53-66

59. Drecktrah D, Knodler LA, Howe D, Steele-Mortimer O: Salmonella trafficking is defined by continuous dynamic interactions with the endolysossomal system. Traffic 2007, 8:212-225.

60. Yu X-J, McGourty K, Liu M, Unsworth KE, Holden DW: pH Sensing by intracellular Salmonella induces effector translocation. Science 2010, 328:1040-1043.

61. Boucrot E, Henry T, Borg JP, Gorvel JP, Mèresse S: The intracellular fate of Salmonella depends on the recruitment of kinesin. Science 2005, 308:1174-1178.

62. Deiwick J, Salcedo SP, Boucrot E, Gilliland SM, Henry T, Petermann N, Waterman SR, Gorvel JP, Holden DW, Méresse S: The translocated Salmonella effector proteins SseF and SseG interact and are required to establish an intracellular replication niche. Infect Immun 2006 , 74:6965-6972.

63. Brumell JH, Goosney DL, Finlay BB: SifA, a Type III secreted effector of Salmonella Typhimurium, directs Salmonella-induced filament (Sif) formation along microtubules. Traffic 2002, 3:407-415.

64. Kuhle V, Hensel M: SseF and SseG are translocated effectors of the type III secretion system of Salmonella pathogenicity island 2 that modulate aggregation of endosomal compartments. Cell Microbiol 2002, 4:813-824.

65. Smith AC, Cirulis JT, Casanova JE, Scidmore MA, Brumell JH: Interaction of the Salmonella-containing vacuole with the endocytic recycling system. J Biol Chem 2005, 280:24634-24641.

66. Ohlson MB, Fluhr K, Birmingham CL, Brumell JH, Miller SI: SseJ deacylase activity by Salmonella enterica Serovar Typhimurium promotes virulence in mice. Infect Immun 2005, 73:6249-6259.

67. Miao EA, Brittnacher AM, Haraga A, Jeng RL, Welch MD, Miller SI: Salmonella effectors translocated across the vacuolar membrane interact with the actin cytoskeleton. Mol Microbiol 2003, 48:401-415.

68. Méresse S, Unsworth KE, Habermann A, Griffiths G, Fang F, MartínezLorenzo MJ, Waterman SR, Gorvel JP, Holden DW: Remodelling of the actin cytoskeleton is essential for replication of intravacuolar Salmonella. Cell Microbiol 2001, 3:567-577.

69. Gallois A, Klein JR, Allen LAH, Jones BD, Nauseef WM: Salmonella pathogenicity island 2-encoded Type III Secretion System mediates exclusion of NADPH oxidase assembly from the phagosomal membrane. J Immunol 2001, 166:5741-5748.

70. Chakravortty D, Hansen-Wester I, Hensel H: Salmonella Pathogenicity Island 2 mediates protection of intracellular Salmonella from reactive nitrogen intermediates. J Exp Med 2002, 195:1155-1166.

71. Fink SL, Cookson BT: Pyroptosis and host cell death responses during Salmonella infection. Cell Microbiol 2007, 9:2562-2570.

72. Paesold G, Guiney DG, Eckmann L, Kagnoff MF: Genes in the Salmonella pathogenicity island 2 and the Salmonella virulence plasmid are essential for Salmonella-induced apoptosis in intestinal epithelial cells. Cell Microbiol 2002, 4:771-781.

73. Gal-Mor O, Gibson DL, Baluta D, Vallance BA, Finlay BB: A novel secretion pathway of Salmonella enterica acts as an antivirulence modulator during Salmonellosis. PLoS Pathog 2008, 4:e1000036.

74. Singer M, Sansonetti PJ: IL-8 is a key chemokine regulating neutrophil recruitment in a new mouse model of Shigella-induced colitis. J Immun 2004, 173:4197-4206.

75. Guichon A, Hersh D, Smith MR, Zychlinsky A: Structure-function analysis of the Shigella virulence factor IpaB. J Bacteriol 2001, 183:1269-1276.

76. Schroeder NG, Hilbi $\mathrm{H}$ : Cholesterol is required to trigger caspase-1 activation and macrophage apoptosis after phagosomal escape of Shigella. Cell Microbiol 2007, 9:265-278. 
77. Suzuki T, Franchi L, Toma C, Ashida H, Ogawa M, Yoshikawa Y, Mimuro H, Inohara N, Sasakawa C, Nuñez G: Differential regulation of caspase-1 activation, pyroptosis, and autophagy via Ipaf and ASC in Shigellainfected macrophages. PLoS Pathog 2007, 3:e111.

78. Way SS, Borczuk AC, Dominitz R, Goldberg MB: An essential role for gamma interferon in innate resistance to Shigella flexneri infection. Infect Immun 1998, 66:1342-1348.

79. Rohde JR, Breitkreutz A, Chenal A, Sansonetti PJ, Parsot C: Type III secretion effectors of the IpaH family are E3 ubiquitin ligases. Cell Host Microbe 2007, 1:77-83

80. Ogawa MY, Handa Y, Ashida H, Suzuki M, Sasakawa C: The versatility of Shigella effectors. Nat Rev Microbiol 2008, 6:11-16.

doi:10.1186/1757-4749-2-8

Cite this article as: Reis and Horn: Enteropathogenic Escherichia coli, Samonella, Shigella and Yersinia: cellular aspects of host-bacteria interactions in enteric diseases. Gut Pathogens 2010 2:8.

\section{Submit your next manuscript to BioMed Central} and take full advantage of:

- Convenient online submission

- Thorough peer review

- No space constraints or color figure charges

- Immediate publication on acceptance

- Inclusion in PubMed, CAS, Scopus and Google Scholar

- Research which is freely available for redistribution

Submit your manuscript at www.biomedcentral.com/submit
C Biomed Central 